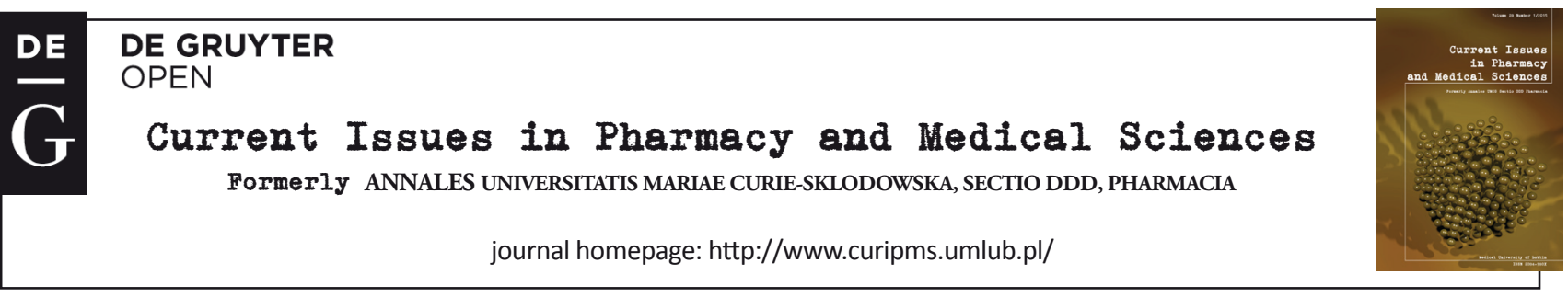

\title{
The international normalized ratio (INR) as seen in a population of patients with atrial fibrillation and cerebral infarction undergoing long-term treatment with vitamin $K$ antagonists
}

\author{
Anna Szczepańska-Szerej ${ }^{1 *}$, Magdalena Wojtan $^{1}$, Beata Szajnoga ${ }^{2}$ \\ ${ }^{1}$ Stroke Unit, Department of Neurology, Medical University of Lublin, Jaczewskiego 8, 20-954 Lublin, Poland \\ ${ }^{2}$ Stroke Unit, Neurology Department of the John Paul II Autonomous Public Voivodeship Hospital, 22-410 Zamosc, Poland
}

\begin{tabular}{l} 
ARTICLE INFO \\
\hline Received 12 October 2015 \\
Accepted 27 November 2015
\end{tabular}

\section{Keywords:}

atrial fibrillation,

cerebral infarction,

international normalized ratio

(INR),

vitamin $\mathrm{K}$ antagonists.

\begin{abstract}
It is estimated that nearly $20 \%$ of all cerebral infarctions in the total population are the result of a complication of atrial fibrillation (AF). While oral anticoagulation with vitamin $\mathrm{K}$ antagonists (AVKs) substantially reduces this risk, this requires regular monitoring of the international normalized ratio (INR) in order to achieve therapeutic levels $(2,0-3,0)$. The aim of this study was to evaluate a group at high risk of cerebral infarction, among patients with AF undergoing long-term treatment with VKAs, taking into account the significance of therapeutic INR values. The analysed group consisted of 90 acute ischaemic stroke patients with paroxysmal or chronic "non-valvular" AF, receiving treatment with VKAs. As a result of the study, therapeutic INR values $(\geq 2)$ were seen in thirty-five of these individuals $(38,8 \%)$, while $55(61,2 \%)$ showed non-therapeutic INR values. Moreover, there were no differences in demographics, vascular risk factors, biochemical and morphological blood parameters, mean CHA2DS2-VASc score and TOAST classification between either of the two groups. Furthermore, no additional factor that would increase their risk of cerebral infarction during the adequate treatment with VKAs was found. However, patients with non-therapeutic INR values had a statistically significantly higher frequency of concomitant moderate pathology of the bicuspid valve, $\mathrm{p}<0.05$. Hence, a lack of proper control of INR can proved to be particularly dangerous for this subgroup of patients. Hence, this is a group with an elevated risk of cerebral infarction and therefore requires special oversight of VKA treatment or NOA treatment.
\end{abstract}

\section{INTRODUCTION}

According to the definition given by the World Health Organization (WHO), stroke is a sudden onset of focal or global disturbance in cerebral function, lasting more than 24 hours, the cause of which is exclusively of vascular origin. More than $80 \%$ of all strokes are ischaemic, with varied or complex aetiology. The determined risk factors of stroke include a group of unmodifiable factors, these being age, sex or race. These factors also include a large group of modifiable factors, wherein particular attention is given to the 'Big Five', i.e. hypertension, atrial fibrillation, diabetes, tobacco smoking and lack of exercise.

\footnotetext{
* Corresponding author

e-mail: aszerej@poczta.onet.pl

tel: +48817244720
}

In clinical practice, atrial $\mathrm{AF}$ is one of the most common arrhythmias, occurring in about $3-5 \%$ of the population. Indeed, it is estimated that nearly $20 \%$ of cerebral infarctions in the total population are a complication of AF; this percentage increases with age, and after the age of 80 , reaches about $30 \%$ [13]. The ischemic stroke rate of patients with $\mathrm{AF}$ is $4-5 \%$ per year [15], five times the general rate [4]. VKAs, alongside new oral anticoagulants, are currently common standards in the treatment of patients with $\mathrm{AF}$, and it is estimated that about $0.8-2.0 \%$ of the population is treated in this manner. A meta-analysis of randomized clinical trials, conducted by Hartman et al. , found that warfarin reduced the risk of cerebral infarction by $64 \%$ and $38 \%$, with respect to a placebo and acetylsalicylic acid (ASA) administration, respectively [7]. However, despite the effectiveness and 
benefits of VKAs, this therapy also has significant limitations. These are associated with high individual variation in the effect of the drug and the need for regular monitoring of the international normalized ratio (INR) in order to achieve therapeutic levels (2.0-3.0). Moreover, VKAs interact with many other drugs. In addition, their metabolism is affected by foods and by liver dysfunction [3].

On the basis of numerous studies, it is estimated that about $30-40 \%$ of patients taking VKAs suffer a cerebral infarction during treatment. In patients who continue the treatment, the INR remains within the therapeutic range in only half [6]. The window is narrow, as an INR under 2 does not provide adequate protection against cerebral infarction, and when the INR is over 3 , there is a dangerous increase in the risk of haemorrhage. Moreover, a therapeutic INR value does not always prevent stroke. In clinical practice, there are many cases of stroke occurring despite therapeutic INR values, or INR exceeding the upper limit of recommended values [2].

The aim of this study was to evaluate a group at high risk of cerebral infarction among patients with AF undergoing long-term treatment with VKAs, taking into account the significance of therapeutic INR values.

\section{MATERIAL AND METHODS}

The study was conducted on patients with cerebral infarction hospitalized at the Stroke Unit, Neurological Department of Autonomous Public Clinical Hospital No. 4, in Lublin and the Stroke Unit, Neurology Department of the John Paul II Autonomous Public Voivodeship Hospital, in Zamosc, in the years 2011-2013.

The group analysed consisted of 90 patients with nonvalvular paroxysmal or chronic AF, receiving long-term treatment with VKAs, who had been admitted to the stroke units named above within the first 24 hours of the stroke. Patients with an artificial heart valve and severe mitral stenosis were not included in the study. The group was divided into two sub-groups according to INR value:

- Group I - 55 patients $-\mathrm{INR}<2$,

- Group II - 35 patients - INR $\geq 2$.

Diagnosis of cerebral infarction was based on the result of a CT scan of the head performed immediately upon admission. The clinical characterization of the groups took into account other modifiable and unmodifiable risk factors for brain stroke: age, sex, hypertension, diabetes, moderate valve insufficiency, kidney failure, heart failure, ischaemic heart disease, infection within 2 weeks before the stroke, history of past stroke or transient ischaemic attack (TIA), as well as peripheral vascular disease, nicotine dependence (past or present), and associated additional treatment.

In addition, in all patients, the risk of cerebral infarction was assessed according to the CHA2DS2-VASc score. Herein, the maximum score is 9 points and each letter corresponds to a different condition and number of points (presence or absence): $\mathrm{C}$ - congestive heart failure (1 pt.); $\mathrm{H}$ - hypertension (1 pt.); $\mathrm{A}$ - age $\geq 75$ (2 pts.); $\mathrm{D}$ - diabetes mellitus (1 pt.); $\mathrm{S}-$ history of stroke or TIA (2 pts.); V (vascular disease) - ischaemic heart disease, peripheral vascular disease, aortic sclerosis, or history of heart attack
(1 pt.); A - age 65-74 (1 pt); Sc (sex category) - female sex (1 pt) [12].

The patients were also evaluated using the OCSP (Oxfordshire Community Stroke Project) classification. This takes into account the location of the infarct on the basis of the clinical syndromes: TACI - total anterior circulation infarct, PACI - partial anterior circulation infarct; LACI - lacunar circulation infarct, or POCI - posterior circulation infarct [1].

Statistical analysis was performed using Student's t-test, the Mann-Whitney U test and the chi-squared test. The level of significance was set at $\mathrm{p}<0.05$.

\section{RESULTS}

The mean value for the INR was $1.35(\min -0,96$, $\max -$ $1,88)$ in group I and 3,69 ( $\min -2.07, \max -8.42)$ in group II. Moreover, the INR on admission was within therapeutic range (2-3) in only $19(21,1 \%)$ of the subjects. Additionally, 16 patients $(17,7 \%)$ had INR values $\geq 3$.

There were no statistically significant differences between groups in terms of sex and age (Tab. 1).

Table 1. Demographic data of the evaluated groups

\begin{tabular}{|c|c|c|c|}
\hline & $\begin{array}{c}\text { Group I, N=55 } \\
\text { ( }(\%)\end{array}$ & $\begin{array}{c}\text { Group II, N=35 } \\
\text { N (\%) }\end{array}$ & P \\
\hline Female & $33(60)$ & $18(51.4)$ & n.s. \\
\hline Age & $\begin{array}{c}\text { Group I, N=55 } \\
\text { mean (SD) }\end{array}$ & $\begin{array}{c}\text { Group II, N=35 } \\
\text { mean (SD) }\end{array}$ & \\
\hline $74.7(10.3)$ & $72.4(8.7)$ & n.s. \\
\hline
\end{tabular}

The groups did not differ in terms of frequency of concomitant hypertension, diabetes, type of AF (chronic or paroxysmal), kidney failure, heart failure, ischemic heart disease, peripheral vascular disease, history of stroke/TIA, or history of nicotine dependence. There was also no statistically significant difference in the mean CHA2DS2-VASc score between the two groups. However, in the group I patients, there was a statistically significantly higher frequency of concomitant pathology of the bicuspid valve (a moderate degree of stenosis or insufficiency), $\mathrm{p}<0.05$.

Table 2. Risk factors for cerebral infarction, accompanying treatment and CHA2DS2-VASc score in the evaluated groups

\begin{tabular}{|l|c|c|c|}
\hline & $\begin{array}{c}\text { Group I, N=55 } \\
\text { N (\%) }\end{array}$ & $\begin{array}{c}\text { Group II, N=35 } \\
\text { N (\%) }\end{array}$ & P \\
\hline Hypertension & $48(87.3)$ & $26(74.3)$ & n.s. \\
\hline Type 2 diabetes & $21(38.2)$ & $11(31.4)$ & n.s. \\
\hline Chronic atrial fibrillation & $40(72.7)$ & $23(65.7)$ & n.s. \\
\hline Valve insufficiency & $13(23.6)$ & $2(5.7)$ & $<0.05$ \\
\hline Kidney failure & $6(10.9)$ & $3(8.6)$ & n.s. \\
\hline Heart failure & $15(27.3)$ & $7(20.0)$ & n.s. \\
\hline History of stroke/TIA & $19(34.5)$ & $12(34.3)$ & n.s. \\
\hline Ischaemic heart disease & $20(36.4)$ & $12(34.3)$ & n.s. \\
\hline Peripheral vascular disease & $2(3.6)$ & $1(2.9)$ & n.s. \\
\hline Current nicotine dependence & $7(12.7)$ & $4(12.1)$ & n.s. \\
\hline Infection on admission & $8(14.5)$ & $5(14.3)$ & n.s. \\
\hline Statins & $28(50.9)$ & $18(51.4)$ & n.s. \\
\hline Anti-hypertensive drugs & $47(85.5)$ & $23(65.7)$ & $<0.05$ \\
\hline & Group I & Group II & \\
\hline CHA2DS2-VASc score & $4.6(1.7)$ & $4.1(1.7)$ & n.s. \\
\hline
\end{tabular}


In addition, the patients in group I were more frequently treated with anti-hypertensive drugs $(\mathrm{p}<0.05)$ (Tab. 2). No differences were found between the two groups in the biochemical and morphological blood parameters tested within 24 hours of the stroke (Tab. 3).

Table 3. Selected biochemical and morphological blood parameters tested within 24 hours of the stroke

\begin{tabular}{|l|c|c|c|}
\hline \multicolumn{1}{|c|}{ Parameter } & $\begin{array}{c}\text { Group I, N=55 } \\
\text { mean (SD) }\end{array}$ & $\begin{array}{c}\text { Group II, N=35 } \\
\text { mean (SD) }\end{array}$ & p \\
\hline Glucose (mg/dl) & $132.7(61.0)$ & $143.3(181.6)$ & n.s. \\
\hline Total cholesterol (mg/dl) & $156.0(40.8)$ & $158.3(38.4)$ & n.s. \\
\hline LDL cholesterol (mg/dl) & $93.6(32.7)$ & $100.0(30.1)$ & n.s. \\
\hline HDL cholesterol (mg/dl) & $44.7(13.6)$ & $39.7(11.7)$ & n.s. \\
\hline Triglycerides (mg/dl) & $98.6(50.1)$ & $108.5(57.7)$ & n.s. \\
\hline CRP (mg/l) & $15.7(21.3)$ & $16.1(21.3)$ & n.s. \\
\hline Fibrinogen (g/l) & $3.84(1.15)$ & $4.3(1.8)$ & n.s. \\
\hline Creatinine (mg/dl) & $1.1(1.0)$ & $1.0(0.3)$ & n.s. \\
\hline Urea (mg/dl) & $42.7(18.1)$ & $43.4(18.6)$ & n.s. \\
\hline ASPAT (U/L) & $27.4(9.6)$ & $30.1(16.8)$ & n.s. \\
\hline ALAT (U/L) & $24.6(13.3)$ & $21.4(7.8)$ & n.s. \\
\hline Bilirubin (mg/dl) & $1.0(0.5)$ & $1.0(0.6)$ & n.s. \\
\hline Leukocytes (1,000/ul) & $8.5(3.3)$ & $13.6(18.9)$ & n.s. \\
\hline Haematocrit (\%) & $39.5(5.7)$ & $41.3(4.3)$ & n.s. \\
\hline Platelets ( $\times 1,000 \mathrm{cells} / \mathrm{ul})$ & $213.7(66.6)$ & $218.8(85.2)$ & n.s. \\
\hline
\end{tabular}

The two groups also did not differ in terms of the clinical evaluation of the size and location of the infarct according to the OCSP classification (Tab. 4).

Table 4. Comparison of the groups according to the OCSP classification

\begin{tabular}{|c|c|c|c|c|}
\hline & $\mathrm{n}$ & $\begin{array}{c}\text { Group I, N=55 } \\
\mathrm{n}(\%)\end{array}$ & $\begin{array}{c}\text { Group II, N=35 } \\
\mathrm{n}(\%)\end{array}$ & $\mathrm{P}$ \\
\hline TACI & 18 & $13(23.6)$ & $5(14.3)$ & \\
\cline { 1 - 4 } PACI & 51 & $28(50.9)$ & $23(65.7)$ & \multirow{2}{*}{ n.s. } \\
\cline { 1 - 4 } LACI & 6 & $5(9.1)$ & $1(2.9)$ & \\
\cline { 1 - 4 } POCI & 15 & $9(16.4)$ & $6(17.4)$ & \\
\hline
\end{tabular}

\section{DISCUSSION}

Atrial fibrillation is an independent variable and the most common cause of cardioembolic cerebral infarction. What is more, over $70 \%$ of patients with this arrhythmia have concomitant cardiovascular disease, mainly including hypertension, left ventricular hypertrophy, valvular defects, diabetes and cardiomyopathy. Yet, the risk of cerebral infarction during the course of atrial fibrillation is not the same in all patients. For this reason, the CHA2DS2-VASc scale is used in these patients to assess the risk of ischemic stroke. It is not known, however, whether the risk of ischemic stroke according to this scale is correlated with INR value, although it might be presumed that effective prophylactic treatment may require higher INR values in patients with AF and with more risk factors for ischaemic stroke. Therefore, we expected that patients in the group with therapeutic INR or higher would have a higher mean CHA2DS2-VASc score, but in fact this value was slightly lower than in the group of patients with non-therapeutic INR. Pujol Lereis et al. [14] compared CHADS2 scores (the original, simpler form of the
CHA2DS2-VASc scale) in patients with AF and ischemic stroke who were receiving proper VKA treatment, with a group of patients who were only taking antiplatelet treatment for stroke prevention. The result of this study was that the scores in these two groups did not differ significantly, This also indirectly suggests that CHADS2 or CHA2DS2-VASc scores are not an additional risk factor for cerebral infarction in patients with therapeutic INR.

Theoretically, patients who had a stroke while receiving VKAs could had other potential etiologies for stroke [5]. Thus, it is particularly important to search for additional stroke etiologies in these cases. For example, in a study by Kim et al. [10] on a group of 55 patients with AF and INR $\geq 2$, the values for CHADS2 /CHA2DS2-VASc scores were found to be correlated with increased risk of stroke in smokers with past ischaemic stroke or TIA.

In our study, individual consideration of all main stroke risk factors and the biochemical and morphological blood parameters had been performed. No one additional factor that would increase the risk of cerebral infarction during adequate treatment with VKAs has been found. Moreover, the clinical form of the completed stroke was the same in both evaluated groups. Patients with non-therapeutic INR more frequently took anti-hypertensive drugs, but this observation does not appear to be significant. No such correlation has been described in the literature.

Moderate bicuspid and aortal valve dysfunction are not included in the CHA2DS2-VASc score and are not indications for anticoagulation treatment. Observational studies provide information that the risk for stroke in people with mitral valve prolapsed or mitral annual calcification is low ( $<1 \%$ annually) $[7,11]$. According to the latest recommendations by the American Heart Association and the American Stroke Association [9], individuals with moderate bicuspid valve insufficiency should take antiplatelet drugs indefinitely. Moreover, anticoagulation may be considered when the left atrium is enlarged $\geq 55 \mathrm{~mm}$ according to echocardiography [16]. Concomitance of AF and valve insufficiency may potentially increase the risk of cardiac embolism, but such patients, due to their AF, should be treated with oral anticoagulants.

Among the patients in our study, we found concomitant mild valve dysfunction four times more frequently in patients with non-therapeutic INR. A lack of proper control of INR proved particularly dangerous for this subgroup of patients. Therefore it seems that particular emphasis should be placed on proper prophylactic therapy in AF patients with concomitant moderate bicuspid valve insufficiency. What is more, where proper control of INR is not possible, it is essential to consider treatment with novel oral anticoagulants (NOA).

\section{CONCLUSIONS}

1. Patients with AF and therapeutic INR need not to have a higher mean CHA2DS2-VASc score and seem to not to have additional factors that would increase their risk of cerebral infarction during treatment with VKAs. Regardless of this observation, individual evaluation of each case is necessary. 
2. Failure to properly control the INR significantly increases the risk of cerebral infarction, particularly in patients with concomitant moderate mitral insufficiency. This is a group with an elevated risk of cerebral infarction, and, therefore, requires special oversight of VKA treatment or NOA treatment.

\section{REFERENCES}

1. Bamford J, at al.: Classification and natural history of clinically identifiable subtypes of cerebral infarction. Lancet, 337,1521-1526,1993.

2. Conolly SJ.: Benefit of oral anticoagulant over antiplatelet therapy in atrial fibrillation depends on the quality of international normalized ratio control achieved by centres and countries as measured by time in therapeutic range. Circulation, 118, 2029-2037, 2008.

3. Crystal E, Connolly SJ: Role of oral anticoagulation in management of atrial fibrillation. Heart 20,813-817, 2004.

4. Garcia DA, Hylek EM.: Antithrombotic therapy in atrial fibrillation. Clin Geriatr Med, 22, 155-156, 2006.

5. Hart RG, at al.: Cardioembolic vs. noncardioembolic strokes in atrial fibrillation: frequency and effect of antithrombotic agents in the stroke prevention in atrial fibrillation studies. Cedrebrovasc Dis, 10, 39-43, 2000.

6. Hart RG, Benavente O, McBride R, Pearce LA: Antithrombotic therapy to prevent stroke in patients with atrial fibrillation: a metaanalysis. Ann Intern Med, 131,492-501, 1999.

7. Hart RG, Halperin JL: Atrial fibrillation and stroke: concepts and controversies. Stroke 32, 803-808, 2001.
8. Hayek E, Griffin B: Mitral valve prolapse: old beliefs yield to new knowledge. Cleve Clin J Med.69:889-896,2002.

9. Kernan WN, at al.: Guidelines for the prevention of stroke in patients with stroke and transient ischemic attack: a guideline for healthcare professionals from the American Heart Association/American Stroke Association. Stroke, 45, 2160-2236, 2014.

10. Kim YD, at al.: Factors associated with ischemic stroke on therapeutic anticoagulation in patients with nonvalvular atrial fibrillation. Yonsei Med J, 56, 410-417, 2015.

11. Kohsaka $S$, at al.: Impact of mitral annular calcification on cardiovascular events in a multiethnic community: the Northern Manhattan Study. JACC Cardiovasc Imaging. 1, 617-623, 2008.

12. Lip GY, at al.: Refining clinical risk stratification for predicting stroke and thromboembolism in atrial fibrillation using a novel risk factor-based approach: the EuroHeart Survey on Atrial Fibrillation. Chest, 137, 263-272, 2010.

13. Lip GY, at al.: ABC of atrial fibrillation. Aethiology, pathophysiology and clinical features. BMI 311,1425-1428,1995.

14. Pujol Leris $\mathrm{W}$, at al.: Ischemic stroke in patients with atrial fibrillation receiving oral anticoagulation. J Neurol Sci, 334, 139-142, 2013.

15. Stramba-Badiale M.: Atriall fibrillation subtypes, risk of stroke, and antithrombotic therapy. Eur Heart J 29, 840-842, 2008.

16. Whitlock R, at al.: Antithrombotic and thrombolytic therapy for valvular disease: Antithrombotic Therapy and Prevention of Thrombosis, 9th ed: American College of Chest Physicians EvidenceBased Clinical Practice Guidelines. Chest, 141:576-600, 2012. 